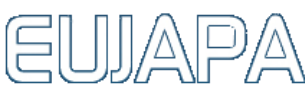

Article

\section{A Qualitative Exploration of Factors Influencing Physical Activity Behaviour for Individuals with Parkinson's Disease Using the Social Ecological Model}

\author{
Bradley MacCosham ${ }^{*}$, Evan Webb², Wahid Hamidi ${ }^{3}$, Jessica Oey ${ }^{4}$, and Francois Gravelle ${ }^{5}$ \\ Received: $30^{\text {th }}$ January 2020; Accepted: $30^{\text {th }}$ October 2020; Published: $8^{\text {th }}$ April 2021
}

\begin{abstract}
Physical activity (PA) can benefit individuals with Parkinson's disease (PD), however, many individuals tend to be sedentary. This qualitative study explored factors influencing PA behaviour for individuals with PD using the social ecological model. Twelve individuals with PD took part in semi-structured interviews. Data were thematically analysed. Results suggest that individuals with PD experience several constraining and facilitating factors to PA behaviour. Intrapersonal constraints revolved around uncertainties that PA is beneficial for individuals with PD, a lack of interest in available PA programs, and disease-specific issues whereas intrapersonal facilitators included prior experiences of enjoyment in PA, seeing improvements, and wanting to maintain independence. Interpersonal constraints related to lacking social support and perceived stigma whereas interpersonal facilitators were, passionate PA program staff, and being active with similar others. Environmental constraints pertained to PA programs failing to adapt program activities, lack of time, and transportation accommodations, whereas environmental facilitators were exposure to non-traditional PA programs, access to resources on PA, and accessibility to community PA programs. Findings highlight the need to address factors influencing PA behaviour for individuals with PD.
\end{abstract}

Keywords: constraints, facilitators, qualitative, long-term illness, barriers

\section{Introduction}

Parkinson's disease (PD) is a neurodegenerative disease which affects dopamineproducing brain cells and causes movement disorders such as bradykinesia, muscular rigidity, postural instability, and tremors (Chaudhuri, Healy, \& Schapira, 2006; Chenoweth, Sheriff, McAnally, \& Tait, 2013; Horstink et al., 2006). PD is one of the most common movement disorders and currently has no cure (Ceravolo, Pagni, Tognoni, \& Bonuccelli, 2012), however PD patients can manage their symptoms through regular physical activity (PA) as long-term management of the disease can be challenging solely through pharmacological treatments (Connolly \& Lang, 2014; Kessler \& Rezak, 2007; Rascol et al., 2003; Tetrud, 2004; Ward \& Robertson, 2004).

PA is a critical aspect of health and chronic disease prevention (Bassuk \& Manson, 2005; Schmitz et al., 2005). Individuals with PD have been known to experience improvements in motor functioning (LaHue, Comella \& Tanner, 2016; Lauzè, Daneault, \& Duval, 2016) and 
quality of life through PA participation (Crizzle \& Newhouse, 2012). However, disease progression and motor symptoms associated with PD such as stooped posture, difficulties with gait, freezing, and postural instability (Boonstra, van der Kooij, Munneke \& Bloem, 2008) make it difficult for individuals with PD to perform routine tasks like walking, bending, and turning (Rezak, 2007). As a result, individuals with PD tend to be sedentary (van Nimwegan et al., 2011) and in turn increase their risk of acquiring cardiovascular disease, diabetes, cognitive impairments, osteoporosis, and depression (Wallèn, Franzèn, Nero, \& Hagströmer, 2015). It is therefore beneficial for individuals with PD to take part in PA pursuits to maintain the health, mobility, and independence that can be lost as a result of disease progression (Schrag, Hovris, Morley, Quinn, \& Jahanshahi, 2006).

A variety of factors discouraging PA participation for individuals with PD have been uncovered in previous research. These include lack of time, fear of falling (Ellis et al., 2013), low self-efficacy, struggling with the diagnosis, lack of support from healthcare professionals, and disease specific issues (Ellis et al., 2011; Khalil, Nazzal, \& Al-Sheyab, 2016; Ravanek \& Schneider, 2009). In contrast, several factors have also been known to facilitate PA behaviour for individuals with PD such as wanting to maintain independence, receiving social support and encouragement from family and exercise professionals, being active among peers, and seeing improvements in daily functioning (Crizzle \& Newhouse, 2012; Khalil et al., 2016).

Moreover, applying ecological models has become standard for designing communitybased PA interventions, developing environmental and policy strategies, and targeting multiple sectors of society such as health care and transportation (Bornstein, Pate, \& Pratt, 2009; Sallis \& Owen, 2015). The social ecological model allows for a deeper analysis of factors influencing PA behaviour as it identifies multilevel factors of influence within personal, social, and environmental domains (Sallis \& Owen, 2015). As such, the social ecological model is a suitable framework for conceptualizing constraining and facilitating factors influencing PA behaviour (Carlson et al., 2012; Orlowski, 2016; Saelens et al., 2012; Sallis \& Owen, 2015).

Intrapersonal, interpersonal, and environmental factors work together to influence PA behaviour, but represent three very different sources of influence (Sallis \& Owen, 2015). Intrapersonal factors consist of individual characteristics such as knowledge, attitudes, behaviour, beliefs, abilities, self-efficacy, and previous life experiences (Sallis \& Owen, 2015). Interpersonal factors have to do with social networks and social support systems such as family and friendships (Sallis \& Owen, 2015). Lastly, the broader category of environmental factors centres on institutions, opportunities within communities, transportation services, convenience and accessibility, healthcare resources and available information, as well as physical structures (Sallis \& Owen, 2015).

Although factors influencing PA behaviour for individuals with PD have been identified, research in this area, and particularly from a social ecological approach within a qualitative Canadian-based context does not exist. By using this approach, this study will make a unique contribution to research which can in turn help develop targeted PA promotion interventions for individuals with PD. Previous research has also not addressed intrapersonal, interpersonal and environmental factors influencing PA behaviour for individuals with PD within the same study. Considering all three types of factors will provide insight on the importance of each group of factors as well the impact of the interaction between factors. This study is also different in that the data explored the history of participant's experiences with PA behaviour prior to their diagnosis with PD and prior to their current involvement in a desired PA program. This approach helped identify changes in PA behaviour pre-diagnosis and post-diagnosis, as well as understanding participants' 
path towards committing to a specific PA program. Thus, the purpose of this study was to qualitatively explore factors influencing PA behaviour for individuals with PD using the social ecological model.

\section{Materials and Methods}

\section{Participants}

Participants of this study were recruited from the Boxing 4 Health program and NeuroLogic Physiotherapy clinic located in Ottawa, Canada. Purposeful and convenience sampling were employed to access participants in this study. Purposeful sampling allowed the primary researcher to recruit knowledgeable individuals who have had first-hand experienced with the phenomenon under investigation (Creswell \& Plano Clark, 2011). Convenience sampling allowed the primary researcher to recruit participants that were in geographic proximity (Etikan, Musa, \& Alkassim, 2016). A purposive and convenience sample of 12 ( 5 females, 7 males) individuals participated in this study, as this was the point in which data saturation was reached.

Boxing 4 Health is a community-based boxing program with the intent of having individuals with PD benefit from a structured PA program. NeuroLogic often refers patients with PD to the Boxing 4 Health program. Each participant recruited from the physiotherapy clinic had been a part of the Boxing 4 Health program at some point in time. Eligibility criteria included being diagnosed with PD by a physician, at least 18 years of age, and capable of engaging in PA on a regular basis. The recruitment process was facilitated by the owners or trainers of the Boxing 4 Health program and therapists at the NeuroLogic clinic who forwarded our recruitment letter to members of Boxing 4 Health and clinic patients. Those interested in participating then contacted the primary researcher and interviews were scheduled at the participants' earliest convenience. Of the 12 participants, 10 were recruited from Boxing 4 Health and 2 were recruited from NeuroLogic. Written consent was obtained before undertaking the interviews.

Qualitative research often has small sample sizes in exchange for large amounts of rich data to gain a good grasp on participants' perceptions without the need to generalize results (Creswell, 2007; Maxwell, 2013). Malterud, Dirk Siersma and Guassora (2016) address the challenges of sampling in the context of qualitative research through the concept of information power. According to the authors, information power is based on the aim of the study, its specificity, the use of established theory, the quality of the dialogue, and the analysis strategy. The aim of the present study is considered to be narrow as the focus is on a specific population in a precise PA program designed to accommodate individuals diagnosed with PD. Therefore, specificity of experiences and knowledge related to participation in PA among the participants included in the sample is quite narrow. The social ecological model provides a sound theoretical framework that enhances the information power of this research in that it is a solid framework to analyse data. Engagement in data collection was carefully planned to provide honest communication between the researcher and participants allowing for co-construction of results and conclusions. Data analysis allowed to uncover relevant information related to the aim of the study, therefore, a smaller number of participants was retained. Participants recruited for this study were aged between 61 and 73 years with a length of PD diagnosis between 1 and 15 years met the eligibility criteria required for this study. Table 1 presents the participants demographic information. Participants' primary source of PA was their engagement in the Boxing 4 Health program. 
Table 1. Participant characteristics

\begin{tabular}{llllll}
\hline ID Code & Gender & Age & Years with PD & Months engaged in B4H & Avg. sessions per week \\
\hline PD1 & Female & 61 & 1 & 7 & 2 \\
PD2 & Female & 62 & 3 & 6 & 3 \\
PD3 & Male & 71 & 1.5 & 5 & 1 \\
PD4 & Male & 64 & 8.5 & 4 & 3 \\
PD5 & Male & 65 & 11 & 1 & 2 \\
PD6 & Female & 73 & 1.5 & 4 & 2 \\
PD7 & Male & 70 & 8 & 5 & 2 \\
PD8 & Male & 72 & 15 & 4 & 3 \\
PD9 & Female & 71 & 2 & 8 & 2 \\
PD10 & Male & 71 & 5 & 1 & 2 \\
PD11 & Male & 67 & 8 & 12 & 3 \\
PD12 & Female & 53 & 13 & 3 & 3 \\
\hline
\end{tabular}

$\mathrm{PD}=$ Parkinson's Disease, $\mathrm{B} 4 \mathrm{H}=$ Boxing 4 Health, Avg. = average

\section{Data collection}

This study was approved by the Office of Research Ethics and Integrity in Health Sciences from University of Ottawa. The qualitative approach was utilized as it seeks to understand processes in which experiences and behaviours take place. In addition, understanding how these processes led to or caused certain behaviours cannot always be understood through the use of predetermined responses (Maxwell, 2004a, 2004b; Miles \& Huberman, 1994). The semi-structured format allowed participants to share experiences and perceptions both pertaining to, and external from, the questions listed on the interview schedule. Interviews were held in the participants' homes, coffee shops in public, and the Boxing 4 Health gym after training sessions. Interviews were recorded using a digital voice recorder and lasted 43 minutes on average.

Interviews were conducted by the first researcher. During the interviews, retrospective information was gathered on participant's experiences in PA prior to their diagnosis and after receiving their diagnosis. With regards to post-diagnosis, participants were asked to elaborate on their experiences by seeking out and engaging in PA, general PA programs, and PA programs adapted for persons with PD. Since there were no standard qualitative instruments looking at the concepts under investigation, this study developed its own semistructured interview guide. Data collection was carried out simultaneously with data analysis to inform and augment how information would be collected throughout the study. Some of the first questions included on the interview were: 'Can you talk to me about your history in physical activity prior to your diagnosis?' and 'Can you talk to me about your involvement in physical activity after your diagnosis?' Additionally, the semi-structured interview guide was framed to have participants address intrapersonal (e.g., what type of personal factors prevented or promoted your pursuit of PA?), interpersonal (e.g., what type of social factors prevented or promoted your pursuit of PA?), and environmental (e.g., what other factors prevented or promoted your pursuit of PA?) factors within the social ecological model framework. However, the interview guide was dynamic and evolved with more specific questions and probes addressing some of the social ecological factors added later.

\section{Data analysis}

Processes for ensuring reliability and validity in qualitative research were utilized to increase the credibility of the findings (Grbich, 2013). The researcher (B.M) performed a bracketing interview to separate their personal experiences and assumptions from their interpretations participants' narratives thus helping to address potential bias. Then, the researchers (B.M., F.G) reflected on their initial understandings of emerging themes by 
journaling their thoughts while reading transcripts for the first time. This also helped the researchers (B.M., F.G) further develop and modify questions in the interview guide to gather more information on emerging themes. The reviewing process has been done on an ongoing fashion during the research process. Data collection, transcription and initial coding was ongoing during the research process, allowing researchers to share views as the research evolved. As researchers' (B.M., E.W., W.H., O.J., F.G) views evolved with the unveiling of results it was possible to reach consensus amongst members of the research team. Lastly, participants were given the opportunity to read their transcript and provide clarifications as a part of the member checking process. Member checking increased the validity of the data in an effort to ensure accuracy and reliability.

Interviews were transcribed verbatim. Data underwent thematic analysis (Grbich, 2013). The first step of the analysis process began with the researchers (B.M., F.G) going over each transcript line by line, reading and re-reading while comparing different narratives until the researchers (B.M., F.G) were comfortable with the information. The researchers (B.M., E.W., W.H., O.J., F.G) underlined key segments of participant narratives while writing descriptive comments alongside them. This allowed the data to reveal ideas as well as behavioural patterns, actions and events that were relevant to the purpose and theoretical framework applied in this study. The key segments of text then underwent deductive coding and were guided by the factors found within the social ecological model (i.e., intrapersonal, interpersonal, environmental). Key segments of text that represented the codes were then grouped with other similar segments of text. Analysis revealed a number constraining and facilitating intrapersonal, interpersonal and environmental factors influencing PA behaviour for individuals with PD. Each factor is presented as a theme with some containing their own set of sub-themes.

Credibility and authenticity of results were addressed through different strategies, namely triangulation, member checking, engagement in data collection, researcher reflexivity, and thick descriptions of the results (Merriam, 2009). Based on Merriam's point of view triangulation of results was performed by using a variety of investigating sources such as literature review and discussion with specialists in the field. Researchers (B.M., E.W., W.H., O.J., F.G) were invited to critically reflect namely upon possible biases, theoretical assumptions through a bracketing interview followed by team discussions. Member checking was ensured not only by having participants react to the transcript of their interviews but also to certain interpretations of the data collected. Adequate engagement in data collection allowed researchers to assess variation and particularities of data.

\section{Results}

\section{Intrapersonal Factors}

For intrapersonal constraints, participants stated how various disease-specific issues associated with PD challenged their ability to participate in PA programs (Table 2). For instance, participants reported being intimidated by committing to a PA program several times per week because of their limited energy and fatigue. Participants also claimed to experience motor-related issues such as difficulties with coordination. A participant stated "we have limited energy. So, to commit yourself to a program two or three times a week may be a lot... [We] may be intimidated because [we've] lost some physical coordination and strength" (PD4).

Furthermore, responses from the participants indicated that the time it took to experience relief provided by medications for treating their PD and their associated side effects physically encumbered their ability to partake in any form of PA. A participant claimed, "My medication [can wear off] so my balance would be off, my strength wouldn't 
be as strong, my speed would certainly slow down... so I have to choose the right time in my day [to be active]" (PD12).

Table 2. Intrapersonal factors influencing PA behaviour for individuals with PD

\begin{tabular}{ll}
\hline Intrapersonal constraints & Intrapersonal facilitators \\
\hline Lack of motivation & Prior experiences of enjoyment in PA \\
Uncertain if PA is beneficial for PD & Seeing improvements \\
Disease-specific issues & -Improving daily functioning \\
-Physical limitations & Wanting to maintain independence \\
-Fatigue & -Wanting to engage in activities before \\
-Unpredictability of medications & disease progresses \\
Limited knowledge on appropriate PA \& PA & \\
programs & \\
-Lack of awareness on PA programs for PD & \\
-Lack of awareness on PA guidelines for PD & \\
Lack of interest in available PA/PA programs & \\
-Lack of variety in programs and ability to & \\
adapt programs & \\
-Lack of understanding on programs & \\
\hline
\end{tabular}

Participants were also constrained in their ability to pursue PA opportunities after being diagnosed with PD because of their limited knowledge on the benefits of PA and limited knowledge of available PA programs for individuals with PD in their community. One participant stated, "I didn't know at that time that [PA] was particularly good for Parkinson's" (PD4). When asked about taking part in other general programs available in their community after being diagnosed with PD, such as cycling or dancing, the same participant mentioned, "I don't think any of those things are organized for Parkinson's" (PD4). The same participant was also asked about PA programs other than boxing that have recently emerged for individuals with PD such as Tai Chi to which he responded, "Tai Chi seems to confuse me I'm not sure how that one works" (PD4). Thus, the participant was unfamiliar with certain types of PA programs offered for individuals with PD and did he seem to understand them. Furthermore, the participant mentioned that he did not consider other programs as viable options for PA. Respondents also explained that some of the activities and programs in their community for individuals with PD simply did not appeal to them when they were informed of these. It was described that, “...[healthcare professionals] say there is a positive relationship between if you go dancing and dealing with your Parkinson's symptoms... but it doesn't appeal to me" (PD3).

Intrapersonal facilitators to PA behaviour pertained to improvements in physical capabilities. For instance, as a result of continued participation in PA programs such as Boxing 4 Health, participants eventually experienced physical improvements (e.g., fine motor skills) in themselves and in other members of the PA program. Experiencing physical improvements appeared to serve as a motivating factor for continued participation in the program. It was explained by a participant that:

[The improvements began] when I started exercising more [and] doing more activities. I started to realize 'hey, there really is some benefit here.' There's one example I had to [pick up a set of keys] three or four years ago [and going through keys using my fingers in one hand was something] I couldn't do... Then, I started lifting weights and after [a few] months of lifting [small] weights on each hand I discovered 'hey I had more control over my fingers.' (PD5)

Similarly, another participant described how seeing others in the boxing program improved physically became a motivating factor for members to stay involved in the 
program. She said "we can see that we can improve each time we're [coming to program sessions]... You can see [others in the program] improving over time and that's very beneficial to [motivate us to keep going]" (PD1).

In addition, participants explained that a desire to fight the disease, maintain their physical functioning and prevent further physical deterioration caused by PD provided sources of motivation to take part in general PA and the Boxing 4 Health program:

...the objective [of being active] is to hinder the degenerative process...

[There's] great motivation to continue and to work hard [in the program]... We

have a real good friend that has had Parkinson's for 15 to 20 years and he's

quite challenged and I would rather not spend the rest of my life that way.

(PD3)

Lastly, findings suggested each participant had experiences of enjoyment in PA prior to receiving their diagnosis of PD (Table 2). This perhaps suggests that participants were motivated to stay physically active and seek resources on adapted PA programs after receiving their diagnosis. A participant also stated that being an active individual prior to receiving their diagnosis may have delayed their diagnosis of PD:

I've always been fairly physical. I played high school football and track and hockey. After university, I did triathlons and cross country skied. I think it's always helped me [maintain an active lifestyle after being diagnosed]. It [also] probably delayed my diagnosis of Parkinson's for a few years. (PD4)

\section{Interpersonal Factors}

Participants also experienced a series of interpersonal constraints (Table 3). A lack of promotion and adequate guidance regarding PA from healthcare professionals were among the most notable constraints. One participant mentioned that "[my healthcare professional said] exercise as much as you can. That was the guidelines... It didn't motivate me" (PD3). It was further explained by participants that healthcare professionals tend to rely heavily on prescribing medication without considering other viable options to manage PD such as PA, as mentioned:

[The neurologist] didn't focus so much on exercise. He told me 'you have to exercise.' When you tell them about exercise, they don't take too much consideration of it... I had an appointment for a treatment for a stiff neck and I asked him to reduce my [medication] dosage because I felt it was strong [and] because [I'm more active now] and he didn't do nothing. (PD2)

Since healthcare professionals are a prime source of information for PD patients after receiving their diagnosis, a working knowledge of PA opportunities would be particularly useful for those treating individuals with PD. As explained by this participant "physicians [are] the first people who know about the diagnosis, so they should also provide the information about the [physical activity] services available related to the disease" (PD6).

Table 3. Interpersonal factors influencing PA behaviour for individuals with PD

\begin{tabular}{ll}
\hline Interpersonal constraints & Interpersonal facilitators \\
\hline Lack of social support & Participating with others is more enjoyable \\
Lack of guidance from healthcare professionals & Passionate staff \\
-Overreliance on medication & -Staff is empathetic \\
-Healthcare professionals' disbelief in PA & -Staff is committed \\
Perceived social stigma & -Staff created positive environment \\
-Isolating one's self & - Staff is knowledgeable about the disease \\
& - Staff adapts program activities. \\
& Being active with similar others \\
& -Experiencing relatedness \\
\hline
\end{tabular}


Leveraging on previous statements, it was also suggested that healthcare professionals may not be well-equipped for inquiring on PD patients' PA behaviour and lack counselling training on motivating PA behaviour, which is perhaps why participants received limited or very broad guidelines. Participants mentioned that healthcare professionals use survey instruments that tend to focus solely on PD related symptoms while neglecting other lifestyle factors such as previous or current PA behaviour, and nutrition as well (e.g., whether patients are on supplements):

I go to [a clinic] and they [have] a screening questionnaire which goes through each stage of Parkinson's on all of the questions to see what sort of symptoms you have. But it doesn't ask you specifically about exercise. I don't think they're aware if that patient is taking supplements [or] if their patients are doing an exercise program. (PD1)

Furthermore, some respondents reported lacking social support. The presence of social support is useful for participants requiring supervision in the event that they are incapable of navigating their environment due to their physical limitations. In particular, one participant who experiences such physical limitations, stated how lacking social support made her fearful that it would be a safety risk for her to engage in general outdoor PA. This statement also indicates a potential relationship between factors influencing PA behaviour in that lacking social support (interpersonal factor) led to a fear of being active (intrapersonal factor):

... I'm scared of walking around in the street... [Having] someone who you can exercise with [would be helpful]... I have a tricycle but for me to go alone on my tricycle it's very hard. I can do it but if I get stuck some place [it would be hard for me to help myself]. (PD2)

It was also reported that some participants perceived social stigma from physical symptoms related to their PD (e.g., tremors) when in public environments. Thus, it was suggested that intrapersonal factors such as tremors may trigger the perception of interpersonal factors such as perceived stigma. As such, participants sometimes avoided engaging in general PA such as walking in the street to prevent others from noticing their symptoms. A participant claimed 'I'm not as outgoing as I once was [probably because] the disease is awkward in public. People tend to stare at you when you're walking in the street, hand-quivering sort of thing. It's almost to the point of embarrassing' (PD11).

Moreover, some participants expressed that program staff at Boxing 4 Health was a facilitator. Many expressed that the presence of passionate staff was a motivating factor for continued participation. Having committed and empathetic staff that can create a positive environment and adapt activities to participants' needs contributed to their desire to stay engaged in the Boxing 4 Health program. For instance, it was mentioned that 'the most important thing I think is that [the staff are] very caring and human beings [...] That has a lot of merit, and most of all it [makes me want to stay] active' (PD2). It was also stated that:

[The staff] know each and every person's flaws and weaknesses. Like sometimes I have a hard time hitting the heavy bag so they'll stand up with gloves on themselves and I tend to hit the gloves [better] as opposed to the heavy bag... They alter that try to alleviate that so it works out quite well individually. (PD11)

Engaging in a structured PA program, such as Boxing 4 Health, with individuals who share common experiences allowed participants to find social support with similar others in 
the program. Members engaging in the same program described how they had formed a community where 'everybody is in the same boat. They're your peers... Everybody basically has a tremor as well. It's much more comfortable at the boxing group.' (PD11). Participants therefore became comfortable being active in a group of similar others and developed a sense of belonging and relatedness with other program members:

There's this community and we come from all different walks of life. We may have never had anything in common to draw us together as a group, but we all have this one thing in common and I enjoy everybody else's company. (PD3)

Another participant emphasized on perceiving the Boxing 4 Health program as an opportune way to discuss with other individuals with PD, which is not something that she has in other areas of her life:

I don't have other areas of my life where there are other people with [PD] that I can talk to... The group is developing into more [of a] social support [group] ... You don't usually exercise with other people with [PD]. (PD1)

\section{Environmental Factors}

One environmental constraint for participants engaging in PA programs outside of Boxing 4 Health was a lack of time. Participants described that when they took part in other PA programs before joining Boxing 4 Health, it was time consuming not only in terms of the duration of the program sessions but also to prepare for the program session. One participant explained the challenges associated with joining an adapted swimming program and how it led to his eventual withdrawal from the program:

You arrive at two o'clock for a session. In the winter time... you've got to get in [the dressing room] and change into your bathing suit, wait for the people in the pool to get out, do your exercises, go in the shower, change, it just became a hassle. (PD10)

Transportation was also identified as an issue for participants engaged in the Boxing 4 Health program, as some have had their driver's license revoked due to their PD. Thus, having to arrange for other forms of transportation to get to and from the program sessions is challenging and seen as unreliable at times:

... Getting [to the program sessions] I have to [accommodate driving] and make an appointment [with the transportation company] and it's got to coincide with the [program session time] and [the drivers are] normally late. [They're] not reliable. Just coordinating myself and finding a way to get there was a bit of an obstacle. (PD10)

Another environmental constraint mentioned by participants included the fact that the variety of activities offered when engaged in programs prior to their commitment to Boxing 4 Health were lacking to the point where they became redundant and made participants feel lethargic. A participant described their experience with programs incorporating routine PA and exercises (e.g., getting up from a chair) to increase participants' physical abilities as boring. It is also described that, despite staff dividing members of the program into low and high functioning groups, it remained unappealing:

There's a city run exercise [program that] do a routine of exercises that are targeted towards [PD]... But, with that I found it's exactly the same exercise, exactly the same instructor, [and the] same audience. It became really boring and I talked to the instructor one time and she said "you've got to adjust for the capabilities of the group. You have people who are reasonably fit and can do the exercises at a moderate pace. You have others who are at the other end of the scale who are barely able to get out of a chair." They did split the group so 
that beginners were in one group and people who are more capable in another group, but even with that I found it boring. (PD10)

Among environmental facilitating factors, participants stated that having access to various resources (e.g., books, internet, media, and seminars) on the benefits of PA for individuals with PD after receiving their diagnosis of PD prompted them to seek options for increasing their PA behaviour (Table 4). One participant explained how her desire to increase the intensity of her exercise was initiated after having read on how PA can benefit individuals with PD such as improving their motor and cognitive skills. Incidentally, understanding the benefits of PA for individuals with PD also motivated them to maintain their independence by offsetting the symptoms of their disease through PA. A participant stated 'I had read about how exercise can help with improving motor skills, maintaining motor skills, and cognitive skills, and I wanted to increase the intensity of my exercise so that I could maybe help prevent deterioration' (PD1).

Table 4 Environmental factors influencing PA behaviour for individuals with PD

\begin{tabular}{ll}
\hline Environmental constraints & Environmental facilitators \\
\hline Staff does not adapt general PA programs for & Exposure to non-traditional PA opportunities \\
persons with PD & Access to resources on PA/PA programs \\
-Lack of variety in the program & -Reading how PA benefits PD in books and \\
Lack of time & the internet \\
-Schedule conflicts with appointments & -Attending seminars \\
-Program takes up a lot of time & PA/PA programs are accessible \\
Transportation & -Activities can be done in any environment \\
-Inability to drive & -Transportation can be easily arranged to the \\
-Difficult to find driving accommodations & program \\
\hline
\end{tabular}

Another facilitator to committing to the Boxing 4 Health program was accessibility to the programs. For instance, Boxing 4 Health offers a transportation service while other participants take it upon themselves to arrange transportation to and from program sessions, as stated, "I have arranged [transportation] to and from program sessions. It picks me up and brings me back home" (PD2). Additionally, given that the Boxing 4 Health activities take place indoors, it was seen as convenient as this keeps adverse weather from inhibiting participation. A Participant explained that 'boxing seems to be convenient and you can do it indoors' (PD4).

The last environmental facilitator described by participants was being exposed to nontraditional PA programs for individuals with PD, such as the Boxing 4 Health program. Being encouraged to engage in boxing which is considered somewhat outside of the norm for PA programs, personal activity pursuits, as well as participants' comfort zones. As such, this may have sparked curiosity in wanting to inquire and learn more about the program. Gaining more information on the program seemed to encourage participants to seek out the new activity that was previously unknown to them, as described:

[When I first heard about the program] I said, '[boxing is] not something that I followed strongly,' until [a staff member] came to our [support] group session and did a presentation and I said 'well that's something I should try.'...It's a different PA. [I'm] trying something new and I think that's good for [me physically [and] mentally. (PD5)

\section{Discussion}

To maintain the health, mobility, and independence that can be lost as a direct result of PD, it is imperative for individuals with PD to take part in PA activities. This study identified factors influencing PA behaviour for individuals with PD using the social ecological model. 
Results indicated that individuals with PD experienced numerous constraining and facilitating factors to PA behaviour at every level of the social ecological model. Intrapersonal constraints centred around doubts that PA is beneficial for individuals with PD, lack of interest in PA programs, and disease specific issues whereas, intrapersonal facilitators consisted of previous experiences of enjoyment in PA, seeing physical improvements, and the desire to maintain independence. Interpersonal constraints discussed were a lack of social support, and perceived stigma whereas, interpersonal facilitators included passionate PA program staff and participating in PA programs with other individuals with PD. Environmental constraints revolved around PA programs failing to adapt and accommodate program activities for individuals with $\mathrm{PD}$, and lack of time while environmental facilitators highlighted exposure to a non-traditional PA program, access to resources on PA, and accessibility to PA programs.

Several themes in this study are consistent with previous research. For instance, constraining intrapersonal, interpersonal, and environmental factors such as diseasespecific issues, lack of support from healthcare professionals, perceived social stigma, and lack of time (Caap-Ahlgren \& Lannerheim, 2002; Khalil et al., 2016; Ravanek \& Schneider, 2009; Thordardottir, Nilsson, Iwarsson \& Haak, 2014) have been uncovered in the past. Facilitating intrapersonal and interpersonal factors highlighted in this study reported in previous studies were, wanting to maintain one's independence, social support and encouragement from family and program staff, being active among similar others, and seeing improvements in daily functioning (Crizzle \& Newhouse, 2012; Khalil et al., 2016).

New understandings can be derived from this research which extend on previous knowledge when it comes to intrapersonal factors related to disease-specific issues. Previous studies had yet to examine how medications taken by individuals with PD could constrain PA behaviour. It was described by participants how the time of day at which medications were taken prior to PA engagement and the unpredictability of how they will react to the medications can physically encumber them. Specifically, if medications were to wear off, they would lose strength and coordination, making it harder to engage in PA. Another interesting intrapersonal factor preventing PA participation was a lack of knowledge on the benefits of PA for individuals with PD. These individuals may have developed the belief that being physically active is potentially detrimental to those with their condition leading to a hesitance to engage in PA and decreased PA behaviour after receiving one's diagnosis. Strategies for educating individuals on the benefits of PA for individuals with PD, such as providing patients with resources, should be practiced more prevalently, especially by healthcare professionals who are often their main source of information.

Further understandings on interpersonal factors pertaining to lack of support from healthcare professionals also arose from our findings. For instance, it was explained by participants that healthcare professionals may be less equipped for aiding individuals with PD on managing symptoms and issues related to PD through PA compared to physiotherapists and staff from adapted PA programs. As was described, clinicians (e.g., neurologists) use survey instruments to gain insight on patients' PD symptoms that may lack items regarding PA lifestyles and behaviour. As a result, neurologists may overlook discussing disease prevention strategies that involve PA and exercise during clinical visits with PD patients. Some participants reported that their neurologists recommended PA, though in these cases they failed to provide adequate guidance and detailed information regarding appropriate opportunities or programs. The lack of appropriate instrumentation to gather information on PD patients' PA behaviour may be an important factor to consider for improving how PA is promoted by neurologists in the future. 
Research in the past has indicated that patients living with chronic conditions and who receive adequate information on $\mathrm{PA}$ and exercise from physicians are more likely to be active when comparing to those who do not receive similar information (Khalil et al., 2016; Yardley, Donovan-Hall, Francis, \& Todd, 2007). Researchers have also emphasized the importance of physicians having a more significant role when it comes to encouraging individuals with PD to become more active, given the perceived importance of their advice on the benefits of PA (Tomlinson et al., 2012). The findings of this study support the idea that neurologists should have a greater role when it comes to promoting PA for individuals with PD, our findings suggest that neurologists may not have the means to do so in an effective manner. One strategy for better promoting PA behaviour for individuals with PD is to increase referrals to physiotherapists as they have adequate resources and knowledge to guide patients towards appropriate PA opportunities and programs. Having neurologists refer patients to physiotherapists immediately after receiving their diagnosis has been associated with preventing early complications from falls and being sedentary (Keus et al., 2007). Similar conclusions on the lack of referrals to physiotherapists from neurologists are brought up in Khalil et al.'s (2016) study. However, Khalil et al.'s (2016) interpretations regarding physiotherapy referrals stem from the lack of available neurologists. Therefore, this leads to referring patients to physiotherapists to relieve neurologists' workload, while the current study supports neurologists working in tandem with physiotherapist for the benefit of their patients with PD.

Moreover, another important interpersonal constraint to PA was perceived social stigma. Participants of this study explained how they believed that their visible PD symptoms attracted the attention of others and made them feel exposed when engaging in public environments. These findings are consistent with previous research (Caap-Ahlgren \& Lannerheim, 2002; Thordardottir et al., 2014). Also, the importance of social interactions with others in a group PA program and being active with other individuals with the same disease is highlighted here as a significant factor for continued participation in a PA program. These individuals are familiar with the challenges of living with PD and interacting regularly with each other offers an opportunity to feel a sense of belonging and relatedness. In such an environment, participants (and staff) encourage each other and feel as though they can share their experiences with other members in the program.

Another intrapersonal facilitator voiced by participants in this study was seeing improvements in physical functioning. Previous studies have also found this to be a motivating factor to stay engaged in PA (Crizzle \& Newhouse, 2012; O’Brien, Dodd, \& Bilney, 2008). Participants reported recognizing the importance of maintaining their engagement in the boxing program once they noticed physical improvements when performing routine tasks in their daily lives. Eriksson, Arne, and Ahlgren (2013) also bring in a social aspect to this theme explaining that seeing improvements in one's self and in others that they participate with can strengthen their beliefs in their capacity to be successful in managing their PD and in turn motivate them to continue participating in the program.

This study also considers factors influencing PA behaviour for individuals with PD once they are already engaged in a structured PA program. Having staff who can create a positive environment and provide continuous reassurance and support in early stages of participation, as was described as an integral interpersonal facilitator by respondents, can keep participants from withdrawing from the program before they have the opportunity to notice or experience physical improvements (van Nimwegan et al., 2011).

The inability to adapt program activities to the needs of participants or introduce new program activities were included as environmental constraints. Participants explained that PA programs they engaged in prior to Boxing 4 Health often lacked in the variety of activities 
that were offered to the point of them becoming redundant and unappealing. It is therefore imperative to have staff that are knowledgeable about PD so that they can adapt program activities to the symptoms and needs of participants. Adapting program activities and introducing new activities is a strategy provided in this study and one that was proposed by participants. Tailoring programs to individuals is common practice when dealing with those who vary in their level of physical functioning (Sánchez-Lastra, Ayán, Sener \& Cancela, 2020). This not only ensures that participants adapt to the program but can also be the critical element for increasing or decreasing their level of enjoyment and motivation to continue in a PA program (O’Brien et al., 2008).

Although this study sought to identify relationships between factors influencing PA, few relationships were discovered. Only one relationship was highlighted. For instance, it was found that the interpersonal constraint of lacking social support triggered an intrapersonal constraint of fearing for one's safety when being active as those companions could help if the individual is injured or loses the ability to move. As such, it may be more advantageous to focus on developing interventions targeting single factors or single types of factors over multilevel factors. Future research should continue to look at existing relationships between factors and how these influence PA behaviour for individuals with PD. Findings on the relationships between social ecological factors has theoretical importance as it helps us further understand how these facets of the model can be mutually interdependent.

Individuals with this study were all active prior to being diagnosed with PD and continue to be motivated to be active today. Findings of this study therefore appear to align with the concept of self-efficacy (Bandura, 1977). Specifically, participants had active lifestyles prior to being diagnosed with PD and thus, past performance accomplishments (Bandura, 1977) or pleasurable experiences with PA in the past seemed to have an important role in participants desire to carry out a course of action (Bandura, 1977) and pursue or maintain PA participation post-diagnosis. Participants were resilient in finding viable PA opportunities despite facing the setback of being diagnosed with $\mathrm{PD}$, as well as experiencing symptoms related to PD. The qualities of these participants therefore demonstrate that individuals with PD can be self-efficacious and maintain an active lifestyle despite being affected by PD.

Limitations of this study must be acknowledged. The geographical scope of this study is limited as participants were mainly recruited from a program in one municipality. Only two participants were recruited from the NeuroLogic clinic compared to the 10 recruited from Boxing 4 Health. Thus, not as much data were collected pertaining to individuals who are not currently engaged in and struggling to find PA opportunities. The sample included more males than females making an unbalanced distribution of gender, which leads to a sample that is not entirely representative. The qualitative data collection process also relies heavily on participants' memories of past events, behaviours, and perspectives. However, memory can be inaccurate at times and subject to considerable distortion (Leedy \& Ormrod, 2013). For example, it may have been more difficult to recall challenges to engaging in PA for participants who received their diagnosis 15 years ago compared to those who have been recently diagnosed. Consequently, this may have compromised some interpretations made by the researchers during the analysis process and the validity of the findings.

Something that was not looked at in this study was whether participants' experiences with constraints and facilitators were impacted by the amount of time that had passed since they had been diagnosed with their condition. Though data concerning how long it has been since participants were diagnosed was collected, the effect this has on their experience of PA related constraints and facilitators was not examined. It is reasonable to suggest that participants change over time in terms of their psychological relationship with PA in light of 
their health and that those who have spent more time participating with their condition may have developed a more substantial set of coping mechanisms than those who have more recently been diagnosed. Future research should look into this due to the useful knowledge this can provide to theory and practice on how length of time since diagnosis influences participant's experiences of PA. In addition, this study did not utilize the Unified Parkinson's Disease Rating Scale (UPDRS) to determine the level of disability of participants. Rather, the researchers explored the PA experiences for individuals with PD. Using the UPDRS to classify participants would have helped associate specific factors to participants based on their classification of UPDRS.

\section{Perspectives}

The findings of this study provide us with further understandings on factors influencing PA behaviour for individuals with PD. The application of the social ecological model helps us with classifying factors influencing PA behaviour for individuals with PD as well as identifying what types of factors are experienced the most. Participants experienced a greater amount of intrapersonal constraints; however, a particular emphasis was held on healthcare professionals' role in promoting PA as this appeared to be one of the most salient themes. This study is also different than previous research as it identified strategies for promoting PA behaviour for individuals with PD based on participant responses when discussing constraints that they experienced. Future research should examine how the findings generated from this study can be applied to develop and implement interventions designed to initiate and maintain PA behaviour for individuals with PD.

Author affiliations:

1 Affiliation 1; Bradley MacCosham; University of Ottawa 125 University Private Ottawa, Ontario; bmacco67@uottawa.ca

2 Affiliation 2; Evan Webb; University of Ottawa 125 University Private Ottawa, Ontario; ewebbo15@uottawa.ca

3 Affiliation 3; Wahid Hamidi; University of Ottawa 125 University Private Ottawa, Ontario; whamio24@uottawa.ca

4 Affiliation 4; Jessica Oey; University of Ottawa 125 University Private Ottawa, Ontario; joey005@uottawa.ca

5 Affiliation 5; Francois Gravelle; University of Ottawa 125 University Private Ottawa, Ontario; fgravelle@uottawa.ca

* Correspondance: bmacco67@uottawa.ca; Tel.: 613-818-5950

Author Contributions: Conceptualization, B.M, and F.G; Methodology, B.M, W.H, J.O, and F.G; Software, B.M, W.H, and J.O; Validation, B.M, E.W, and F.G; Formal Analysis, B.M, and F.G; Investigation; B.M, and F.G; Resources, B.M, E.W, W.H, J.O, and F.G; Data Curation, B.M, and F.G; Writing-Original Draft Preparation, B.M, E.W, W.H, J.O, and F.G; Writing-Review and Editing, B.M, E.W, W.H, J.O, and F.G; Visualization, B.M, and F.G; Supervision, B.M, and F.G; Project Administration, B.M, and F.G.

Funding: This research received no external funding.

Acknowledgments: None.

Conflicts of Interest: The authors declare no conflict of interest.

\section{References}

Bandura, A. (1977). Self-efficacy: Toward a unifying theory of behavioral change. Pyschology Review, 84, 191-215. https://doi.org/10.1037/0033-295X.84.2.191

Bassuk, S. S., \& Manson, J. E. (2005). Epidemiological evidence for the role of physical activity in reducing risk of type 2 diabetes and cardiovascular disease. Journal of Applied Physiology, 99(3), 1193-1204. https://doi.org/10.1152/japplphysiol.00160.2005

Boonstra, T. A., van der Kooij, H., Munneke, M., \& Bloem, B. R. (2008). Gait disorders and balance disturbances in Parkinson's disease: Clinical update and pathophysiology. Current Opinions in Neurology, 24(4), 461-471. https://doi.org/10.1097/wco.0b013e328305bdaf 
Bornstein, D. B., Pate, R. R., \& Pratt, M. (2009). A review of the national physical activity plans of six countries. Journal of Physical Activity and Health, 6(2), 245-264. https://doi.org/10.1123/jpah.6.s2.s245

Caap-Ahlgren, M., \& Lannerheim, L. (2002). Older Swedish women's experiences of living with symptoms related to Parkinson's disease. Journal of Advanced Nursing, 39(1), 87-95. https://doi.org/10.1046/j.1365-2648.2002.02245.X

Carlson, J. A., Sallis, J. F., Conway, T. L., Saelens, B. E., Frank, L. D., Kerr, J., . . King, A. C. (2012). Interactions between psychosocial and built environment factors in explaining older adult's physical activity. Preventive Medicine, 54(1), 68-73. https://doi.org/10.1016/j.ypmed.2011.10.004

Ceravolo, R., Pagni, C., Tognoni, G., \& Bonuccelli, U. (2012). The epidemiology and clinical manifestations of dysexecutive syndrome in Parkinson's disease. Frontiers in Neurology, 3, 159. https://doi.org/10.3389/fneur.2012.00159

Chaudhuri, K. R., Healy D. G., \& Schapira, A.H. (2006). Excellence NIfC. Non motor symptoms of Parkinson's disease: Diagnosis and management. The Lancet Neurology, 5(3), 235-245. https://doi.org/10.1016/S1474-4422(06)70373-8

Chenoweth, L., Sheriff, J., McAnally, L., \& Tait, F. (2013). Impact of the Parkinson's disease medication protocol program on nurses' knowledge and management of Parkinson's disease medicines in acute and aged care settings. Nurse Education Today, 33(5), 458-464. https://doi.org/10.1016/j.nedt.2012.04.022

Connolly, B. S., \& Lang, A. E. (2014). Pharmacological treatment of Parkinson disease: A review. JAMA, 311(16), 1670-1683. https://doi.org/10.1001/jama.2014.3654

Creswell, J. W. (2007). Qualitative inquiry \& research design: Choosing among five approaches (2nd ed.). Thousand Oaks, CA: SAGE.

Creswell, J. W., \& Plano Clark, L. (2011). Designing and conducting mixed methods research (2nd ed.). Thousand Oaks, CA: SAGE.

Crizzle, A. M., \& Newhouse, I. J. (2012). Themes associated with exercise adherence in persons with Parkinson's disease: A qualitative study. Occupational Therapy in Health Care, 26(2-3), 174-186. https://doi.org/10.3109/07380577.2012.692174

Ellis, T., Boudreau, J. K., DeAngelis, T. R., Brown, L. E., Cavanaugh, J. T., Earhart, G. M., Dibble, L. E. (2013). Barriers to exercise in people with Parkinson disease. Physical Therapy, 93(5), 628-636. https://doi.org/10.2522/ptj.20120279

Ellis, T., Cavanaugh, J. T., Earhart, G. M., Ford, M. P., Foreman, K. B., Fredman, L., Dibble L. E. (2011). Factors associated with exercise behavior in people with Parkinson disease. Physical Therapy, 91(12), 1838-1848. https://doi.org/10.2522/ptj.20100390

Eriksson, B. M., Arne, M., \& Ahlgren, C. (2013). Keep moving to retain the healthy self: The meaning of physical exercise in individuals with Parkinson's disease. Disability and Rehabilitation, 35(26), 2237-2244. https://doi.org/10.3109/09638288.2013.7753.357

Etikan, I., Musa, S., \& Alkassim, R. (2016). Comparison of convenience sampling and purposive sampling. American Journal of Theoretical and Applied Statistics, 5(1), 1-4. https://doi.org/10.11648/j.ajtas.20160501.11

Grbich, C. (2013). Qualitative data analysis: An introduction (2nd ed.). Thousand Oaks, CA: SAGE.

Horstink, M., Tolosa, E., Bonuccelli, U., Deuschl, G., Friedman, A., Kanovsky, J., Larsen, P. (2006). Review of the therapeutic management of Parkinson's disease. Report of a joint task force of the European Federation of Neurological Societies (EFNS) and the Movement Disorder Society-European Section (MDS-ES). Part II: Late (complicated) Parkinson's disease. European Journal of Neurology, 13(11), 1186-1202. https://doi.org/10.1111/j.1468-1331.2006.01548.x

Kessler, A., \& Rezak, M. (2007). Complications of dopaminergic therapy. Disease a month, 53(4), 223-226. https://doi.org/10.1016/j.disamonth.2007.05.004

Keus, S. H., Bloem, B. R., Hendriks, E. J., Bredero-Cohen, A. B., \& Munneke, M. (2007). Evidence-based analysis of physical therapy in Parkinson's disease with recommendations for practice and research. Movement Disorders, 22(4), 451-460. https://doi.org/10.1002/mds.21244 
Khalil, H., Nazzal, M., \& Al-Sheyab, N. (2016). Parkinson's disease in Jordan: Barriers and motivators. Physiotherapy Theory and Practice, 32(7), 509-519. https://doi.org/10.1080/09593985.2016.1219433

Lahue, S., Comella, C., \&Tanner, C. (2016). The best medicine ? The influence of physical activity and inactivity on Parkinson's disease. Movement Disorders, 31(10), 1444-1454.

Lauze, M., Daneault, J., \& Duval, C. (2016). The effects of physical activity in Parkinson's disease. Journal of Parkinson's Disease, 6(4), 685-698. https://doi.org/10.3233/JPD160790

Leedy, P. D., \& Ormrod, J. E. (2013). Practical Research: Planning and Design (10th ed.). New York, NY: Pearson Education Inc.

Malterud K, Siersma VD, Guassora AD (2016). Sample size in qualitative interview studies: Guided by information power. Qualitative Health Research 26(13):1753-1760 https://doi.org/10.1177/1049732315617444

Maxwell, J. A. (2004a). Causal explanation, qualitative research, and scientific inquiry in education. Educational Researcher, 33(2), 3-11. https://doi.org/10.3102/0013189x033002003

Maxwell, J. A. (2004b). Using qualitative methods for causal explanation. Field Methods, 16(3), 243-264. https://doi.org/10.1177/1525822x04266831

Maxwell, J. A. (2013). Qualitative research design: An interactive approach (3rd ed.). Thousand Oaks, CA: SAGE.

Merriam S.B. (2009) Qualitative Research A guide to Design and Implementation. John Wiley \& Sons, inc. San Francisco CA, USA, p. 304.

Miles, M. B., \& Huberman, A. M. (1994). Qualitative data analysis: An expanded sourcebook. Thousand Oaks: SAGE.

O'Brien, M., Dodd, K. J., \& Bilney, B. (2008). A qualitative analysis of a progressive resistance exercise program for people with Parkinson's disease. Disability and Rehabilitation, 30(18), 1350-1357. https://doi.org/10.1080/09638280701614546

Orlowski, M. (2016). Introduction to health behaviors: A guide for managers, practitioners \& educators. Boston, MA: Cengage Learning.

Rascol, O., Payoux, P., Ory, F., Ferreira, J. J., Brefel-Courbon, C., \& Montastuc, J. L. (2003). Limitations of current Parkinson's disease therapy. Annals of Neurology, 53(3), 3-15. https://doi.org/10.1002/ana.10513

Ravenek, M., \& Schneider, M. (2009) Social support for physical activity and perceptions of control in early Parkinson's disease. Disability and Rehabilitation 31: 1925-1936. https://doi.org/10.1080/09638280902850261

Rezak, M. (2007). Current pharmacotherapeutic treatment options in Parkinson's disease. Disease Month, 53, 214-222. https://doi.org/10.1016/j.disamonth.2007.05.002

Saelens, B. E., Sallis, J. F., Frank, L. D., Cain, K. L., Conway, T. L., Chapman, J. E., ... Kerr, J. (2012). Neighborhood environment and psychosocial correlates of adults' physical Activity. Medicine \& Science in Sports \& Exercise, 44(4), 637-646. https://doi.org/10.1249/mss.obo13e318237fe18

Sallis J. F., \& Owens, N. (2015). Ecological models of health behavior. In K. Glanz, F. M. Lewis, \& B. K. Rimer (Eds.), Health Behavior and Health Education: Theory, Research, and Practice, 43-64(5th ed). San Francisco, CA: Jossey-Bass.

Sánchez-Lastra, M.A., Ayán, C., Sener, M., \& Cancela, J.M. (2020). The use of adapted boxing as a rehabilitation strategy in people with diverse health conditions: a systematic review. European Journal of Adapted Physical Activity, 13(1), 6. https://doi.org/10.5507/euj.2020.004

Schmitz, K. H., Holtzman, J., Courneya, K. S., Mâsse, L. C., Duval, S., \& Kane, R. (2005). Controlled physical activity trials in cancer survivors: A systematic review and metaanalysis. Cancer Epidemiology, Biomarkers \& Prevention, 14(7), 1588-1595. https://doi.org/10.1158/1055-9965.EPI-04-0703

Schrag, A., Hovris, A., Morley, D., Quinn, N., \& Jahanshahi, M. (2006). Caregiver-burden in Parkinson's disease is closely associated with psychiatric symptoms, falls, and disability. Parkinsonism \& Related Disorders, 12(1), 35-41.

https://doi.org/10.1016/j.parkreldis.2005.06.011 
Tetrud, J. (2004). Treatment challenges in early stage Parkinson's disease. Neurologic Clinics, 22(3), 19-33. https://doi.org/10.1016/j.ncl.2004.05.002

Thordardottir, B., Nilsson, M. H., Iwarsson, S., \& Haak, M. (2014). You plan, but you never know - participation among people with different levels of severity of Parkinson's disease. Disability and Rehabilitation 36(26), 2216-2224. https://doi.org/10.3109/09638288.2014.898807

Tomlinson, C. L., Patel, S., Meek, C., Clarke, C. E., Stowe, R., Shah, L., ... Sackley, C. M. (2012). Physiotherapy versus placebo or no intervention in Parkinson's disease. Cochrane Database of Systematic Reviews, 8, CDo02817.

https://doi.org/10.1002/14651858.CDo02817.pub2

van Nimwegan, M., Speelman, A. D., Hofman-van Rossum, E. J., Overeem, S., Deeg, D. J. H., Borm, G. F., Munneke, M. (2011). Physical inactivity in Parkinson's disease. Journal of Neurology, 258(12), 2214-2221. https://doi.org/10.1007/s00415-011-60977

Wallèn, M. B., Franzèn, E., Nero, H., \& Hagströmer, M. (2015). Levels and patterns of physical activity and sedentary behavior in elderly people with mild to moderate Parkinson disease. Physical Therapy, 95(8), 1135-1141. https://doi.org/10.2522/ptj.20140374

Ward, C. D., \& Robertson, D. (2004). Rehabilitation in Parkinson's disease. Reviews in Clinical Gerontology, 13(3), 223-239. https://doi.org/10.1017/So959259804001054

Yardley, L., Donovan-Hall, M., Francis, K., \& Todd, C. (2007). Attitudes and beliefs that predict older people's intention to undertake strength and balance training. The Journals of Gerontology Series B: Psychosocial Sciences and Social Sciences, 62(2), 119-125. https://doi.org/10.1093/geronb/62.2.P119

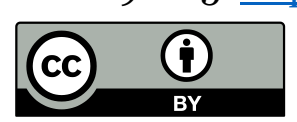

(C) 2021 by the authors. Submitted for possible open access publication under the terms and conditions of the Creative Commons Attribution (CC BY) license (http://creativecommons.org/licenses/by/4.o/). 